\section{Taking out teratoma potential}

\section{By Kai-Jye Lou, Staff Writer}

Researchers at the Stanford University School of Medicine have developed an approach for separating human pluripotent stem cells from teratoma-forming cells in culture. ${ }^{1}$ The group thinks the procedure could help allay tumorigenicity concerns associated with stem cell-derived therapies and plans to use the approach to test existing therapeuticgrade stem cell-derived pancreatic cells and neural progenitors for their teratoma-forming potential.

When researchers differentiate pluripotent of stem cells, a trace number may remain in an undifferentiated state. These undifferentiated cells can form teratomas in animals. ${ }^{2}$ Teratomas are tumors that contain cells derived from all three germ tissue layers.

Indeed, the potential for tumorigenicity has prompted the FDA to require developers of stem cell-derived therapies to run assays and animal studies showing no residual undifferentiated cells and no tumor formation.

Companies have attempted to address the tumorigenicity concerns by developing assays and by tweaking their manufacturing processes, such as using a protracted differentiation protocol to increase the homogeneity of the clinical product.

Assays detect but do not remove the undifferentiated cells, and changes to the manufacturing process do not guarantee the absence of undifferentiated cells in the clinical product.

"There is currently no gold standard for removing teratomaforming cells from differentiated cultures," said Chad Tang, a medical student at Stanford and lead author on the Nature Biotechnology paper.

"We wanted to develop a prospective method to remove the teratoma risk of undifferentiated cells," added Micha Drukker, cocorresponding author on the paper and a postdoctoral scholar at Stanford. "We asked whether we can come up with specific surface marker combinations that could be used to remove teratoma-forming cells prior to transplantation."

The Stanford researchers settled on mAbs as a potential solution. Indeed, previous papers by the Bioprocessing Technology Institute in Singapore showed that mAbs can kill undifferentiated human embryonic stem (ES) cells. ${ }^{3,4}$

Using fluorescence-activated cell sorting (FACS) and antibody libraries, the Stanford team identified a specific marker for undifferentiated human ES cells that they dubbed stage-specific embryonic antigen-5 (SSEA-5).

The group generated a mixed population of differentiated and undifferentiated cells by differentiating human ES cells with retinoic acid for three days. They then used an SSEA-5-targeting $\mathrm{mAb}$ and FACS to sort the population into fractions with high and low SSEA-5 expression.

Mice receiving the cell fraction with low SSEA-5 expression developed smaller teratomas than mice given the fraction with high SSEA-5 expression.

When mice received fractions depleted of cells expressing SSEA-5 and two additional surface markers of pluripotency-CD9 and thymus cell antigen $1 \theta$ (Thy1; CD90) or CD30 and CD200-no animals developed teratomas. Although 3 of the 10 mice developed small nonteratoma growths, follow-up histology studies suggested that these growths originated from committed precursor cells as opposed to pluripotent cells.

According to Drukker, growths stemming from such cell populations should be a lesser cause for concern, if at all, because unlike pluripotent cells, committed precursor cells eventually differentiate into their terminal cell types and stop dividing. With prolonged differentiation times, these precursor cells should disappear from culture, he added.

"Our study identifies a combination of molecules that would enable the prospective removal of cells with teratoma potential from therapeutic preparations derived from pluripotent stem cells," said Drukker.

"We don't need to wait until teratomas form to remove the originating cells," added Tang.

\section{Identify yourself}

The Stanford group thinks its approach could help address the tumorigenicity concerns of stem cell-derived cells. As an added benefit, Tang said the technique could help decrease the time needed to differentiate stem cells in culture, which would speed up the production of therapeutic-grade products.

"It could be a good idea to integrate this approach into the development pipeline at stem cell companies at some point prior to treating patients," Drukker told SciBX. "For example, one could envision taking a sample of the clinical product and then using a panel of these antibodies to confirm that it is devoid of teratoma-forming cells before transplanting the cells into the patient."

"This work represents an important advance, but the feasibility of the mAb-based approach remains to be determined," said Yang 
$\mathrm{Xu}$, a professor in the Division of Biological Sciences at the University of California, San Diego. "Most types of pluripotent stem cellderived cells with potential for therapy will die or be induced to further spontaneous differentiation when dissociated into single cells and sorted by flow cytometry."

Robert Lanza, CSO of Advanced Cell

Technology Inc., said the value of the Stanford group's approach would be product dependent.

"We already have assays with the sensitivity to detect a single, undifferentiated cell from a population of a million cells, and in our clinical trials using stem cell-derived retinal pigment epithelial cells, we only need to transplant a maximum of 200,000 cells. Thus, this antibody approach would probably not be applicable to our RPE cell program. But in clinical settings where one would want to transplant less pure populations of stem cell-derived cells, such as hematopoietic cells, these antibodies could help reduce the risk of teratoma formation," he said.

ACT's human ES cell-derived RPE cells are in Phase I/II testing to treat Stargardt's macular dystrophy and dry age-related macular degeneration (AMD).

Lanza also thinks the approach described in the paper needs to be refined.

"In its current state, even targeting multiple markers didn't seem to remove all the tumor-forming cells in these mixed-cell populations," he told SciBX. "Also, while the approach could help remove some trace number of cells that could be problematic, one will still need to carry out lifetime studies in animals to show that the clinical product does not cause tumors before it could be tested in patients."

"The use of the antibody approach to eliminate the teratoma risk in a cell therapy model would be helpful in validating its potential," added Xu.

Both Drukker and Tang acknowledged that the combination of molecules to target with mAbs will need to be tailored to each stem cell-derived cell therapy to ensure that all the undifferentiated cells are removed without also removing the differentiated cells.

For example, noted Drukker, stem cellderived pancreatic cells express high levels of CD200, so a CD200-targeting mAb would probably need to be swapped out for one that targets another pluripotency surface marker.

Drukker said the group now plans to validate whether the differentiation protocols used to create therapeutic-grade stem cell-derived cells completely differentiate all the cells in culture and eliminate teratoma risk.

"We will utilize current directed protocols to differentiate pluripotent cells into what are now considered therapeutic-grade pancreatic cells and neural progenitors and test whether these populations are safe for transplantation," he told SciBX. "If we find that undifferentiated cells remain, we will test if we can use a combination of antibodies from our panel to remove the teratoma-initiating cells."

Stanford has a pending patent covering the approach. The work is available for licensing.

Lou, K.-J. SciBX 4(33); doi:10.1038/scibx.2011.925

Published online Aug. 25, 2011

\section{REFERENCES}

1. Tang, C. et al. Nat. Biotechnol.; published online Aug. 14, 2011; doi:10.1038/nbt. 1947

Contact: Micha Drukker, Stanford University School of Medicine, Stanford, Calif.

e-mail:dmicha@stanford.edu

Contact: Irving L. Weissman, same affiliation as above e-mail: irv@stanford.edu

2. Herberts, C.A. et al. J. Transl. Med. 9, 29 (2011)

3. Choo, A.B. et al. Stem Cells 26, 1454-1463 (2008)

4. Tan, H.L. et al. Stem Cells 27, 1792-1801 (2009)

\section{COMPANIES AND INSTITUTIONS MENTIONED}

Advanced Cell Technology Inc. (OTCBB:ACTC), Santa Monica, Calif. Bioprocessing Technology Institute, Singapore Stanford University School of Medicine, Stanford, Calif. University of California, San Diego, La Jolla, Calif. 